SLAC-PUB-11781

March 2006

\title{
Emittance Limitation of a Conditioned Beam in a Strong Focusing FEL Undulator*
}

\author{
Z. Huang, G. Stupakov \\ Stanford Linear Accelerator Center, Stanford, CA 94309 \\ S. Reiche \\ University of California at Los Angeles, Los Angeles, CA 90095
}

\begin{abstract}
Various methods have been proposed to condition an electron beam in order to reduce its emittance effect and to improve the short-wavelength free electron laser (FEL) performance. In this paper, we show that beam conditioning does not result in a complete elimination of the emittance effect in an alternating-gradient focusing FEL undulator. Using a one-dimensional model and a three-dimensional simulation code, we derive a criteria for the emittance limitation of a perfectly conditioned beam that depends on the focusing structure.
\end{abstract}

Contributed to the ICFA Beam Dynamics Workshop on the Physics and Applications of High Brightness Electron Beams Erice, Sicily, Italy (October 9-14, 2005)

*Work supported by Department of Energy contracts DE-AC02-76SF00515. 


\section{Introduction}

A primary factor limiting the performance of short-wavelength, high-gain free electron lasers (FELs) is the electron beam transverse emittance. In order to generate transversely coherent radiation with the diffraction limited emittance $\lambda_{r} / 4 \pi$ ( $\lambda_{r}$ being the radiation wavelength), the ideal electron transverse emittance $\varepsilon$ should be less than $\lambda_{r} / 4 \pi$ for the efficient beam-radiation interaction. This requirement is usually not met in the x-ray wavelength region. The spread in the transverse betatron motion degrades the resonant FEL interaction and limits the short-wavelength reaches of an x-ray FEL.

To mitigate this problem, it has been proposed to "condition" an electron beam prior to the undulator by increasing each particle's energy in proportion to the square of its betatron amplitude [1. This conditioning enhances the FEL gain by reducing the axial velocity spread within the electron beam generated over the undulator, due to both energy spread and finite transverse emittances.

The original proposal [1] to condition the beam utilizes a set of cavities in $\mathrm{TM}_{210}$ mode immersed in a focusing lattice. Later approaches to the problem invoke a slow TM waveguide mode internal to the undulator [2, and an energy chirp in the beam in combination with a chromatic delay line [3, 4, 5, 6]. It has also been shown that an attempt to condition the beam on a short distance is accompanied by a head-tail focusing variation which can result in the large effective transverse emittance growth [4]. Although this emittance growth can be avoided [6], the pace of conditioning becomes much slower, and the required length of the conditioner considerably increases. Other proposals are based on using lasers and include Thomson backscattering [], and interaction of the laser with the electron beam in two dedicated undulators [8]. Recently, beam conditioning using nonlinear RF acceleration is also discussed 9 .

In the FEL gain analysis of the original proposal [1, the undulator natural focusing is assumed to confine the electron beam in both transverse directions. This results in the complete elimination of the emittance effect for a perfectly conditioned beam. In this paper, we study a more practical situation when the transverse focusing is provided by alternating-gradient quadrupoles in the undulator, as found in typical x-ray FEL designs. We show that although conditioning eliminates the effect of the average slippage

of electrons relative to the radiation phase, there remain phase oscillations with the period given by the focusing lattice. These phase oscillations can 
affect the FEL performance and impose a limitation on the beam emittance even for a perfectly conditioned beam, albeit this emittance limitation is much relaxed as compared to a unconditioned beam.

\section{Emittance effect in a strong focusing undu- lator}

Although a magnetic undulator can provide natural focusing in both transverse planes [10, the focusing strength is typically too weak for the highenergy electron beam that drives an x-ray FEL. Thus, alternating-gradient quadrupole magnets are inserted in undulator breaks to provide the necessary strong focusing, usually in the form of a FODO lattice (consisting of repetitive focusing-undulator-defocusing-undulator cells). The horizontal betatron motion is given by

$$
\begin{aligned}
& x_{\beta}(z)=\sqrt{2 J_{x} \beta_{x}} \cos \Phi_{x}(z), \\
& p_{x}(z) \equiv \frac{d x_{\beta}}{d z}=-\sqrt{\frac{2 J_{x}}{\beta_{x}}}\left[\sin \Phi_{x}(z)+\alpha_{x} \cos \Phi_{x}(z)\right],
\end{aligned}
$$

where $J_{x}$ is the horizontal action of the electron, $\alpha_{x}$ and $\beta_{x}$ are the Twiss parameters, and $p_{x}$ denotes the angle of the orbit with the $z$ axis. The second term in Eq. (11) is ignored in Ref. 11] under the smooth approximation. Its importance in FEL dynamics and emittance compensation (i.e., conditioning) is pointed out in Ref. [12].

To avoid a large beam size variation in the undulator, the FODO lattice is usually designed to have a small phase advance per cell. In this case, the average betatron wavelength $2 \pi \bar{\beta}$ is much larger than the FODO cell length $L_{c}$. In a first approximation the beta function is constant over the length of the undulator, and the accumulated betatron phase is

$$
\Phi_{x}(z)=\phi_{x}+\int_{0}^{z} \frac{d s}{\beta_{x}(s)} \approx \phi_{x}+\frac{z}{\bar{\beta}},
$$

where $\phi_{x}$ is the initial electron phase. More importantly for the emittance compensation, such a FODO lattice has [12]

$$
\alpha_{x}=-\frac{1}{2} \frac{d \beta_{x}}{d z} \approx \pm 1+O\left(\frac{L_{c}^{2}}{\bar{\beta}^{2}}\right)
$$




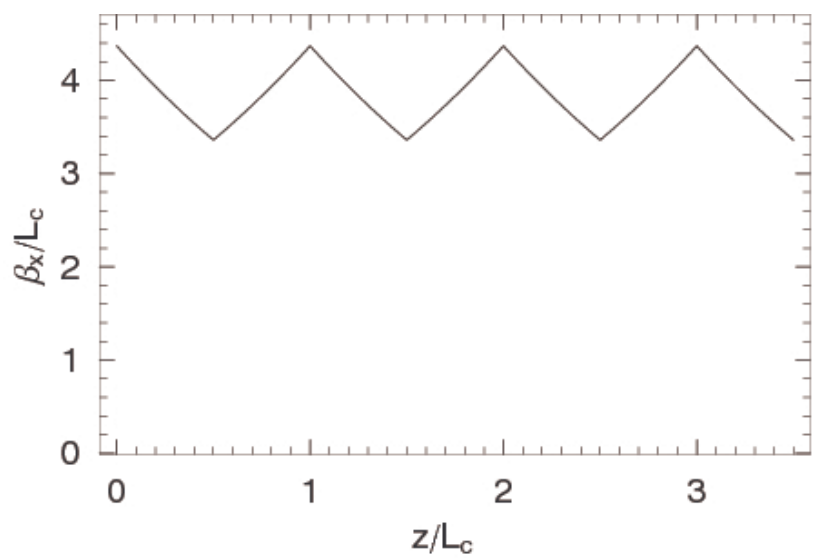

Figure 1: Variation of the beta function along the distance of the lattice for the phase advance per cell of 15 degrees. The derivative $d \beta_{x} / d z$ is close to the values \pm 2 , but the deviation of $\beta_{x}$ from the average value $\bar{\beta}$ is relatively small.

with the sign alternating per half cell. Fig. 1 illustrates the variation of the beta function in a FODO lattice with 15 degrees phase advance per cell.

Under this short-cell-length approximation, the square of the horizontal divergence is

$$
p_{x}^{2} \approx \frac{2 J_{x}}{\bar{\beta}}\left[1 \pm \sin \left(\frac{2 z}{\bar{\beta}}+2 \phi_{x}\right)\right] .
$$

Similarly in the vertical $y$ direction, we have

$$
p_{y}^{2} \approx \frac{2 J_{y}}{\bar{\beta}}\left[1 \mp \sin \left(\frac{2 z}{\bar{\beta}}+2 \phi_{y}\right)\right] .
$$

Here the signs \pm becomes $\mp$ due to the focusing-defocusing asymmetry. We have also assumed that the average beta function is the same in both directions.

The FEL interaction is a resonant phenomenon that depends critically on the evolution of the electron phase relative to the co-propagating radiation field. The so-called ponderomotive phase is defined as $\theta=\left(k_{r}+k_{u}\right) z-$ $c k_{r} \bar{t}$, where $k_{r}=2 \pi / \lambda_{r}$ is the radiation wavenumber, $k_{u}=2 \pi / \lambda_{u}, \lambda_{u}$ is the undulator period, and $\bar{t}$ is the electron arrival time at the location $z$ averaged over the undulator period. The rate of the phase change is

$$
\frac{d \theta}{d z}=\left(k_{r}+k_{u}\right)-\frac{c k_{r}}{v_{z}},
$$


where $v_{z}$ is the undulator-period-averaged longitudinal velocity and is given by

$$
\frac{v_{z}}{c} \approx 1-\frac{1}{2 \gamma^{2}}-\frac{v_{\perp}^{2}}{2}=1-\frac{1+K^{2} / 2}{2 \gamma^{2}}-\frac{p_{x}^{2}+p_{y}^{2}}{2} .
$$

Here $K$ is the undulator parameter and is approximately constant. Its weak dependence on $x$ and $y$ can be neglected as long as the external focusing of the FODO lattice is much stronger than the natural focusing of the undulator. Making use of the resonant condition

$$
k_{r}=\frac{2 \gamma_{r}^{2} k_{u}}{1+K^{2} / 2}
$$

we can write Eq. (6) as

$$
\begin{aligned}
\frac{d \theta}{d z} & =2 k_{u} \frac{\Delta \gamma}{\gamma_{r}}-\frac{k_{r}}{2}\left(p_{x}^{2}+p_{y}^{2}\right) \\
& =2 k_{u} \frac{\Delta \gamma}{\gamma_{r}}-k_{r} \frac{\left(J_{x}+J_{y}\right)}{\bar{\beta}} \\
& -k_{r}\left[ \pm \frac{J_{x}}{\beta} \sin \left(\frac{2 z}{\bar{\beta}}+2 \phi_{x}\right) \mp \frac{J_{y}}{\beta} \sin \left(\frac{2 z}{\bar{\beta}}+2 \phi_{y}\right)\right]
\end{aligned}
$$

Since the last term of Eq. (9) is oscillatory with the FODO lattice period (cell length), the main accumulating effects on the ponderomotive phase are the first two terms. Neglecting this oscillatory term, the phase equation in such a FODO cell is identical to that in a natural focusing undulator [13, as noted in Refs. 12, 14. For a beam with finite energy spread and emittance, the first two terms introduce phase slippage of the electron relative to the radiation and result in the phase spread of the beam. Denoting \langle\rangle as the average over the beam, we have $\left\langle J_{x}\right\rangle=\varepsilon_{x}$ and $\left\langle J_{y}\right\rangle=\varepsilon_{y}$, where $\varepsilon_{x, y}=\varepsilon_{n} / \gamma_{r}$ are the beam transverse emittances. In order to not significantly degrade the FEL performance, the emittance-induced phase spread over one FEL power gain length $L_{G}$ should be less than unity, i.e.,

$$
\frac{2 k_{r} \varepsilon_{n}}{\gamma_{r} \bar{\beta}} L_{G}<1, \quad \text { or } \quad \varepsilon_{n}<\gamma_{r} \frac{\lambda_{r} \bar{\beta}}{4 \pi L_{G}} .
$$

This situation changes if the initial electron energy can be conditioned to its transverse betatron amplitudes such that

$$
2 k_{u} \frac{\Delta \gamma_{0}}{\gamma_{r}}=k_{r} \frac{\left(J_{x}+J_{y}\right)}{\bar{\beta}}
$$


the dominant emittance effect (i.e., the second term in Eq. (9)) is then removed from Eq. (9). Averaging over $J_{x}$ and $J_{y}$, the (transversely) correlated energy spread required to condition a beam with the normalized emittance $\gamma_{r} \varepsilon_{x}=\gamma_{r} \varepsilon_{y}=\varepsilon_{n}$ is

$$
\left\langle\Delta \gamma_{0}\right\rangle_{c}=\frac{\lambda_{u}}{\lambda_{r}} \frac{\varepsilon_{n}}{\bar{\beta}}
$$

However, when the beam is perfectly conditioned to satisfied Eq. (12), the last oscillatory term in Eq. (92) is no longer negligible and can play a limiting role with a large enough emittance. We discuss quantitatively this remaining emittance effect on the FEL performance for a conditioned beam in the following two sections.

\section{1D model of the ponderomotive phase os- cillation}

The ponderomotive phase oscillations in Eq. (9) depend on the transverse variables through $J_{x}, J_{y}, \phi_{x}$ and $\phi_{y}$ and is a three-dimensional (3D) problem. To isolate the oscillation effect and to simplify the problem, we study a heuristic one-dimensional (1D) model with the following phase equation

$$
\frac{d \theta}{d z}=2 k_{u} \delta+\frac{k_{r} \varepsilon_{n}}{\gamma_{r} \bar{\beta}} f(z)
$$

where $\delta=\left(\Delta \gamma-\Delta \gamma_{0}\right) / \gamma_{r}$ is the FEL-induced energy change, and $f(z)$ represents the oscillatory behavior introduced by a conditioned beam in the FODO lattice (with a cell length much smaller than the betatron wavelength), i.e.,

$$
f(z)= \begin{cases}+1 & \text { when }(n-1) L_{c} \leq z<(2 n-1) \frac{L_{c}}{2}, \\ -1 & \text { when }(2 n-1) \frac{L_{c}}{2} \leq z<n L_{c},\end{cases}
$$

and $n=1,2,3 \ldots$ In the absence of the FEL interaction (i.e., when $\delta=0$ ), the phase oscillates between 0 and $\theta_{0}$, with the maximum phase deviation

$$
\theta_{0}=\frac{k_{r} \varepsilon_{n} L_{c}}{2 \gamma_{r} \bar{\beta}}
$$

Using the FEL Pierce parameter $\rho$ [15], we introduce a scaled distance $\tau=2 k_{u} \rho z$ and a scaled energy $\eta=\delta / \rho$. Equation (13) becomes

$$
\frac{d \theta}{d \tau}=\eta+\xi, \quad \text { with } \quad \xi=\frac{k_{r} \varepsilon_{n}}{2 \gamma_{r} k_{u} \rho \bar{\beta}} f(\tau) .
$$


The FEL-induced energy change is

$$
\frac{d \eta}{d \tau}=a e^{i \theta}+\text { complex conjugate }
$$

where $a$ is the slowly-varying radiation field amplitude (properly scaled by $\rho)$. Neglecting any transverse dependence and considering the radiation field at the resonant frequency $c k_{r}$, the $1 \mathrm{D}$ field equation is

$$
\frac{d a}{d \tau}=-\left\langle e^{-i \theta}\right\rangle
$$

This set of coupled equation can be solved by averaging over the fast oscillation if its period is much smaller than the field gain length, similar to the undulator-period averaging procedure for a planar undulator. To illustrate this process, we define

$$
\tilde{\theta}=\theta-\int^{\tau} \xi\left(\tau^{\prime}\right) d \tau^{\prime}
$$

Equation (16) can be written as

$$
\frac{d \tilde{\theta}}{d \tau}=\eta
$$

Equations (17) and (18) become

$$
\begin{aligned}
& \frac{d \eta}{d \tau}=a e^{i \tilde{\theta}} \exp \left(i \int_{0}^{\tau} \xi\left(\tau^{\prime}\right) d \tau^{\prime}\right)+\text { complex conjugate } \\
& \frac{d a}{d \tau}=-\left\langle e^{-i \tilde{\theta}}\right\rangle \exp \left(-i \int_{0}^{\tau} \xi\left(\tau^{\prime}\right) d \tau^{\prime}\right)
\end{aligned}
$$

Treating $\eta, \tilde{\theta}$, and $a$ as slowly-varying variables, we can average Eqs. (21) and (22) over the oscillation period $L_{c}$ to obtain

$$
\begin{aligned}
& \frac{d \eta}{d \tau}=a e^{i \tilde{\theta}} A\left(\theta_{0}\right)+\text { complex conjugate } \\
& \frac{\overline{d a}}{d \tau}=-\left\langle e^{-i \tilde{\theta}}\right\rangle A\left(-\theta_{0}\right)
\end{aligned}
$$




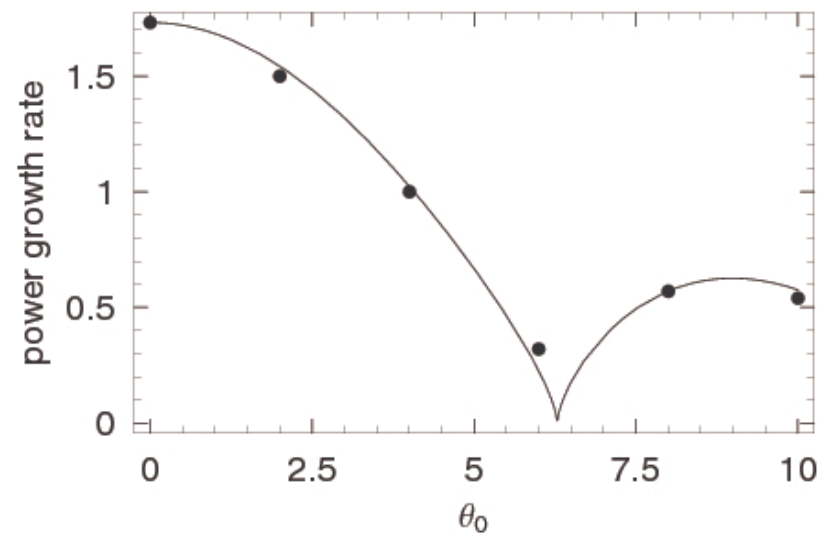

Figure 2: 1D FEL power growth rate under a rapid phase oscillation with the maximum phase deviation $\theta_{0}$. Solid curve is the solution of Eq. 26, and the symbols represent $1 \mathrm{D}$ simulation results.

where

$$
\begin{array}{r}
A\left(\theta_{0}\right)=\int_{0}^{L_{c}} \frac{d z}{L_{c}} \exp \left(i \int_{0}^{\tau} \xi\left(\tau^{\prime}\right) d \tau^{\prime}\right) \\
=\int_{0}^{L_{c}} \frac{d z}{L_{c}} \exp \left(i \theta_{0} \int_{0}^{z} \frac{2 d s}{L_{c}} f(s)\right) .
\end{array}
$$

Equations (20), (23) and (24) are the FODO-cell averaged FEL equations and can be solved with the usual techniques (see, e.g., Ref. 15]). Assuming that $a(\tau) \propto e^{-i \mu \tau}$, we obtain a cubic equation for the complex growth rate $\mu$ :

$$
\mu^{3}=\left|A\left(\theta_{0}\right)\right|^{2} .
$$

The FEL power growth rate $2 \operatorname{Im} \mu=\sqrt{3}\left|A\left(\theta_{0}\right)\right|^{2 / 3}$ versus the maximum phase deviation $\theta_{0}$ is shown in Fig. 2. It agrees well with the $1 \mathrm{D}$ simulation results by solving the original set of Eqs. (16), (17) and (18) for an electron beam without any initial energy spread. The FEL growth rate is degraded when

$$
\theta_{0}=\frac{k_{r} \varepsilon_{n} L}{2 \gamma_{r} \beta}>1 .
$$


Table 1: GENESIS FEL simulation parameters of conditioned beams in a strong focusing undulator.

\begin{tabular}{|llc|}
\hline Parameter & Symbol & Value \\
\hline electron energy & $\gamma_{r} m c^{2}$ & $20 \mathrm{GeV}$ \\
flattop bunch current & $I_{p k}$ & $3 \mathrm{kA}$ \\
transverse norm. emittance & $\varepsilon_{n}$ & 0.1 to $19 \mu \mathrm{m}$ \\
conditioned energy spread & $\left\langle\Delta \gamma_{0}\right\rangle_{c}$ & given by Eq. (12) \\
uncorrelated energy spread & $\sigma_{\gamma_{0}}$ & 0 \\
undulator period & $\lambda_{u}$ & $3 \mathrm{~cm}$ \\
undulator parameter & $K$ & 4.67 \\
average beta function & $\bar{\beta}$ & $4.8 / 9.4 / 19 \mathrm{~m}$ \\
FODO cell length & $L_{c}$ & $2.64 / 5.04 / 9.83 \mathrm{~m}$ \\
FEL wavelength & $\lambda_{r}$ & $1.0 \AA$ \\
\hline
\end{tabular}

\section{3D GENESIS simulations}

The above 1D model shows that when the maximum phase oscillation amplitude exceeds unity, the FEL gain will be degraded as compared to the ideal case. Therefore, for a perfectly conditioned beam that satisfies Eq. (12) in a FODO lattice, the FEL performance will still be affected by the emittanceinduced phase oscillations when the normalized emittance $\varepsilon_{n}$ exceeds a critical emittance given by

$$
\varepsilon_{n}^{c} \equiv \gamma_{r} \frac{\lambda_{r} \bar{\beta}}{\pi L_{c}}
$$

Since this emittance criteria is derived with a heuristic 1D model, we examine it using the 3D FEL code GENESIS [16]. The electron beam and undulator parameters are given in Table 1. A typical FEL power evolution $(P$ versus $z)$ is shown in Fig. 3 (blue dashed curve). The local power growth rate $1 / P(d P / d z)$ is also plotted in the same figure (green solid curve) and is oscillatory with a period equaling to $L_{c} / 2$ as a result of emittance-induced phase oscillations.

We extract the FEL power gain length by averaging a relatively constant $1 / P(d P / d z)$ over many oscillation periods in the GENESIS simulations. The resulting gain length versus the normalized emittance is plotted in Fig. 目 for $\bar{\beta}=19 \mathrm{~m}$ and for three different FODO cell lengths. We also compare the simulation results with the theoretical gain length for a conditioned beam 


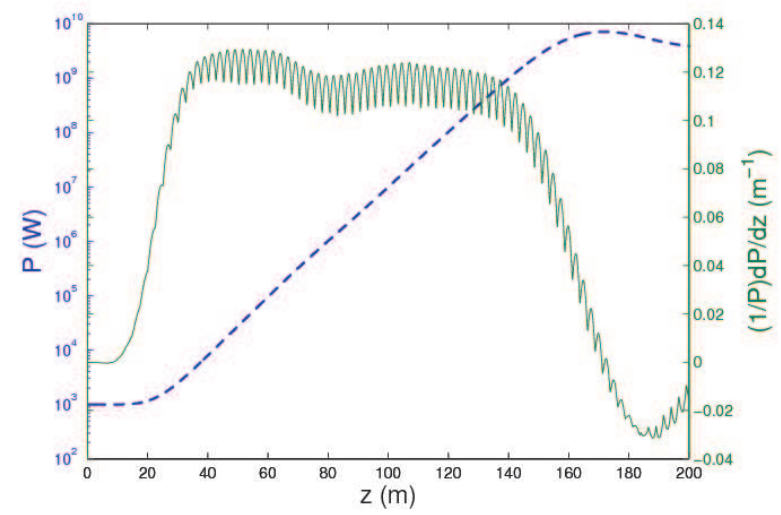

Figure 3: (Color) GENESIS amplifier run of a conditioned beam for $L_{c}=$ $5.04 \mathrm{~m}, \bar{\beta}=19 \mathrm{~m}$ and $\varepsilon_{n}=10 \mu \mathrm{m}$. The blue dashed curve is the FEL power $P$ at $\lambda_{r}=1 \AA$, while the green solid curve is the local growth rate $1 / P(d P / d z)$.

in the absence of any phase oscillation. The latter is obtained by solving the usual 3D eigenmode equation (see, e.g., Ref [17]) for the fundamental Gaussian mode without any energy and angular spreads. As shown in Fig. 4. the simulation results agree with the 3D theory that does not take into account phase oscillations up to the critical emittances predicted by Eq. (28). For emittances exceed these critical values, the gain lengths extracted from simulations start to increase faster than the ideal case.

We have also performed GENESIS simulations by varying the average beta function $\bar{\beta}$ while keeping the same focusing structure (with $L_{c}=2.64 \mathrm{~m}$ ). Figures 5 and $\left[6\right.$ show the gain length comparisons for $L_{c}=2.64 \mathrm{~m}$ at $\bar{\beta}=$ $9.4 \mathrm{~m}$ and $4.8 \mathrm{~m}$, respectively. Combining with Fig. 4, we see that Eq. (28) is reasonably accurate in predicting the critical emittances for different beta functions and FODO lattices.

As the ratio of the average beta function to the cell length becomes too large (e.g., when $\bar{\beta}=39 \mathrm{~m}$ and $L_{c}=2.64 \mathrm{~m}$ or when $\bar{\beta}=19 \mathrm{~m}$ and $L_{c}=1.44 \mathrm{~m}$ ), the gain length extracted from the GENESIS simulation starts to deviate from the 3D theory (without any phase oscillation) at a smaller emittance than that predicted by Eq. (28). A plausible explanation is given as follows. In x-ray FELs the transverse coherence builds up slowly as many higher-order transverse modes are excited by an electron beam with a relatively large emittance. To some degrees a periodic modulation in the electron 


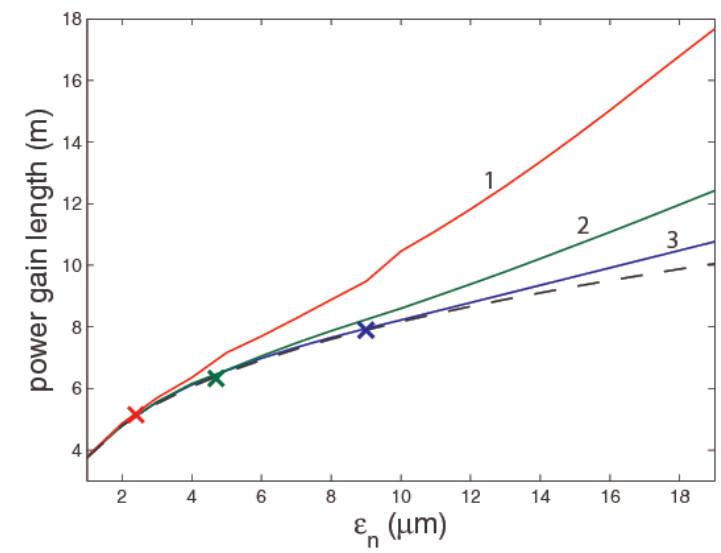

Figure 4: (Color) FEL power gain length from GENESIS simulations at $\bar{\beta}=19 \mathrm{~m}$ for $L_{c}=2.64 \mathrm{~m}$ (blue solid curve 1 ), $L_{c}=5.04 \mathrm{~m}$ (green solid curve 2 ), $L_{c}=9.83 \mathrm{~m}$ (red solid curve 3 ), and from $3 \mathrm{D}$ theory without any phase oscillation (black dashed curve). The colored symbols represent the corresponding critical emittance for each lattice (determined by Eq. (28)) when the phase oscillation is expected to increase the gain length.

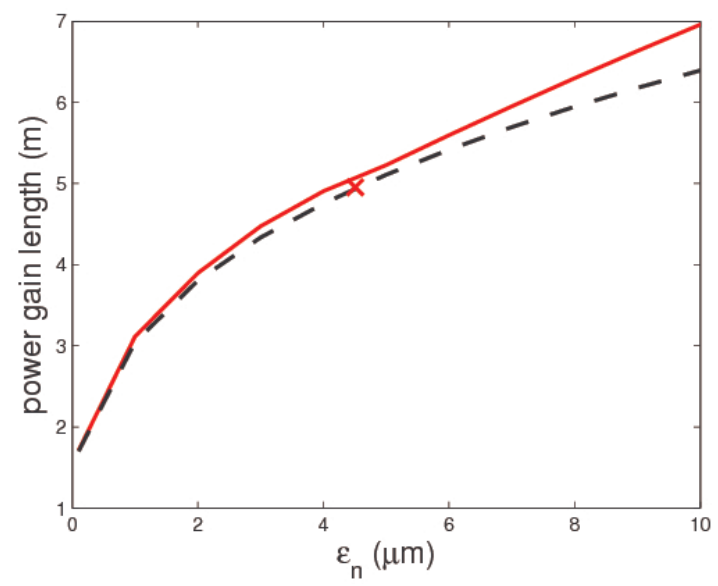

Figure 5: (Color) FEL power gain length from GENESIS simulations at $\bar{\beta}=9.4 \mathrm{~m}$ for $L_{c}=2.64 \mathrm{~m}$ (red solid curve), and from 3D theory without any phase oscillation (black dashed curve). The symbol represents the corresponding critical emittance (determined by Eq. (28)) when the phase oscillation is expected to increase the gain length. 


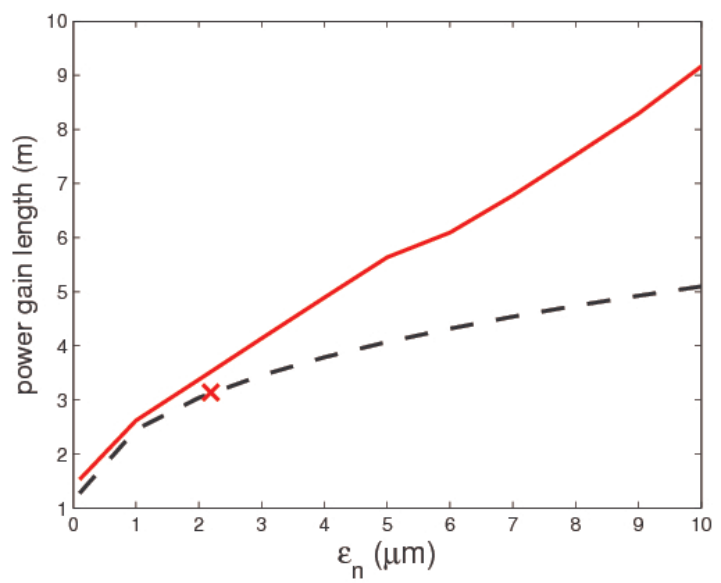

Figure 6: (Color) FEL power gain length from GENESIS simulations at $\bar{\beta}=4.8 \mathrm{~m}$ for $L_{c}=2.64 \mathrm{~m}$ (red solid curve), and from 3D theory without any phase oscillation (black dashed curve). The symbol represents the corresponding critical emittance (determined by Eq. (28)) when the phase oscillation is expected to increase the gain length.

beam size along the undulator is beneficial to the build-up of the transverse coherence (namely higher order modes are suppressed by a varying beam envelope [18]). When the ratio of the beta function to the cell length becomes too large, this higher-order suppression mechanism is not effective since the beam size is almost constant, and the growth of the radiation is contributed from both the fundamental mode as well as higher-order modes. In this case, the comparison between simulations (including higher-order modes) and the theory (for the fundamental mode) is no longer valid.

\section{Summary}

In this paper, we study the FEL performance for a perfectly conditioned beam in a strong focusing undulator consisting of FODO cells. We develop a heuristic 1D model for the emittance-induced phase oscillation and show that the maximum phase deviation should be less than unity in order not to degrade the FEL gain. This criteria limits the maximum emittance that may be conditioned in such a focusing channel and is confirmed by the 3D GENESIS simulations over a reasonably wide parameter range. Therefore, 
instead of the usual emittance criteria for the unconditioned beam as given by Eq. (10), the emittance of the conditioned beam in a FODO lattice with an average beta function $\bar{\beta}$ and a cell length $L_{c}$ must satisfy

$$
\varepsilon_{n}<\gamma_{r} \frac{\lambda_{r} \bar{\beta}}{\pi L_{c}}
$$

That is, the emittance requirement for a conditioned beam is much relaxed but not without any limitation.

\section{Acknowledgments}

We thank A. Zholents for useful discussions. This work was supported by Department of Energy contract DE-AC02-76SF00515.

\section{References}

[1] A. Sessler et al., Phys. Rev. Lett., 68, 309 (1992).

[2] P. Sprangle et al., Nucl. Instrum. Methods A 331 (1993) 6.

[3] N. A. Vinokurov, Nucl. Instrum. Methods A 375 (1996) 264.

[4] P. Emma and G. Stupakov, Phys. Rev. ST Accel. Beams, 6, 030701 (2003).

[5] P. Emma and G. Stupakov G. Proceedings of 9th European Particle Accelerator Conference, p. 503 (2004)

[6] A. Wolski, G. Penn, A. Sessler, and J. Wurtele Phys. Rev. ST Accel. Beams 7, 080701 (2004)

[7] C. B. Schroeder, E. Esarey, and W. P. Leemans Phys. Rev. Lett. 93, $194801(2004)$

[8] A. Zholents, Phys. Rev. ST Accel. Beams 8, 050701 (2005)

[9] G. Stupakov and Z. Huang, in Proc. of the FEL'05, Stanford, CA, USA, p. 176 (2005). 
[10] E. Scharlemann, J. Appl. Phys. 58, 2154 (1985).

[11] L.-H. Yu, C. Huang, D. Li, and S. Krinsky, Phys. Rev. A 51, 813 (1995).

[12] S. Reiche, Nucl. Instrum. Methods A 445, 90 (2000).

[13] In a natural focusing undulator, $\alpha_{x, y}=0$ in Eq. (11), but the transverse variations of the undulator parameter $K$ together with the first term in the bracket of Eq. (11) result in the phase equation $d \theta / d z=$ $2 k_{u} \Delta \gamma / \gamma_{r}-k_{r}\left(J_{x}+J_{y}\right) / \beta_{n}$, where $\beta_{n}=\gamma_{r} \lambda_{u} /(\pi K)$ is the natural focusing beta function. See also Ref. [10].

[14] E. Saldin, E. Schneidmiller, and M. Yurkov, Nucl. Instrum. Methods A 475, 86 (2001).

[15] R. Bonifacio, C. Pellegrini, and L. Narducci, Opt. Commun. 50, 373 (1984).

[16] S. Reiche, Nucl. Instrum. Methods A 429, 243 (1999).

[17] M. Xie, Nucl. Instrum. Methods A 445, 59 (2000).

[18] S. Reiche, Ph. D. dissertation, DESY (1999). 